\title{
El-CID: A filter for Gravitational-wave Electromagnetic Counterpart Identification
}

\author{
Deep Chatterjee, ${ }^{1,2 \star}$ Gautham Narayan, ${ }^{1,2}$ Patrick D. Aleo, ${ }^{1,2}$ Konstantin Malanchev, ${ }^{2,3}$ \\ and Daniel Muthukrishna ${ }^{4}$ \\ ${ }^{1}$ Center for AstroPhysical Surveys, National Center for Supercomputing Applications, Urbana, IL, 61801, USA \\ ${ }^{2}$ Department of Astronomy, University of Illinois at Urbana-Champaign, 1002 West Green Street, Urbana, IL 61801, USA \\ ${ }^{3}$ Lomonosov Moscow State University, Sternberg Astronomical Institute, Universitetsky pr. 13, Moscow, 119234, Russia \\ ${ }^{4}$ Institute of Astronomy, University of Cambridge, Madingley Road, Cambridge CB3 OHA, UK
}

13 October 2021

\begin{abstract}
As gravitational-wave interferometers become more sensitive and probe ever more distant reaches, the number of detected binary neutron star mergers will increase. However, detecting more events farther away with gravitational waves does not guarantee corresponding increase in the number of electromagnetic counterparts of these events. Current and upcoming wide-field surveys that participate in GW follow-up operations will have to contend with distinguishing the kilonova from the ever increasing number of transients they detect, many of which will be consistent with the GW sky-localization. We have developed a novel tool based on a temporal convolutional neural network architecture, trained on sparse early-time photometry and contextual information for Electromagnetic Counterpart Identification (El-CID). The overarching goal for El-CID is to slice through list of new transient candidates that are consistent with the GW sky localization, and determine which sources are consistent with kilonovae, allowing limited target-of-opportunity resources to be used judiciously. In addition to verifying the performance of our algorithm on an extensive testing sample, we validate it on AT2017gfo - the only EM counterpart of a binary neutron star merger discovered to date - and AT2019npv - a supernova that was initially suspected as a counterpart of the gravitationalwave event, GW190814, but was later ruled out after further analysis.
\end{abstract}

Key words: neutron star mergers - gravitational waves - simulations

\section{INTRODUCTION}

While the discovery of gravitational waves (GWs) has been rather recent, (Abbott et al. 2016) the field has moved swiftly since. The discovery rate has increased more than an order of magnitude over the last five years, from three events in the first observing run to more than thirty in the most recent catalog (Abbott et al. 2020a). With the start of the third LIGO/Virgo Collaboration (LVC) observing run, O3, in 2019 GW astrophysics has moved into the public alert era. LVC has created critical infrastructure ${ }^{1}$ to broadcast discovery information in near real-time so that $\mathrm{GW}$ candidates can be followed-up in the electromagnetic (EM) spectrum. Identifying the EM counterpart enables much richer multiwavelength studies of these events, providing much stronger constraints on the evolution of the progenitor system during the kilonova explosion, and the resulting ejecta composition. This, in turn, allows us to understand the role of these events in the broader context of galaxy evolution. Combining EM and GW data may also improve the utility of these events as

\footnotetext{
* E-mail: deep1018@illinois.edu

1 https://emfollow.docs.ligo.org/userguide/
}

standard sirens, (Schutz 1986; Holz \& Hughes 2005) helping to break the degeneracy between their distance and inclination, allowing their use as cosmological probes in the nearby Universe (Abbott et al. 2017b).

The resources expended by the community on multimessenger astrophysics is warranted. The first ever observed binary neutron star (BNS) merger, GW170817, (Abbott et al. 2017a) discovered on August 17, 2017 was followed by a short gamma-ray burst, GRB 170817A, (Goldstein et al. 2017) and the first joint observation of the kilonova (KN), AT 2017gfo across the electromagnetic spectrum ${ }^{2}$ (Abbott et al. 2017c; Arcavi et al. 2017; Coulter et al. 2017; Kasliwal et al. 2017; Lipunov et al. 2017; Soares-Santos et al. 2017; Tanvir et al. 2017; Valenti et al. 2017). Subsequent observations of this one event over the next hours through months, by facilities worldwide revealed that it was the site of heavy element nucleosynthesis. It provided the first observational evidence for the several decade-old hypothesis that compact object mergers can be the progenitors of short GRBs and KNe (Lattimer \& Schramm 1974, 1976; Blinnikov et al. 1984; Li \&

2 There have been reports of potential kilonovae earlier from GRBs, see for example Tanvir et al. (2013) 
Paczyński 1998; Metzger et al. 2010).The wealth of data of GW170817 then reminds us of the promise of the science that is enabled by identifying the EM counterparts of these events. The third observing run reported a total of 56 candidates released publicly. The GWTC-2 catalog (Abbott et al. 2020a) has reported more events than those discovered during real-time analysis. However, despite many advances and significant effort by the community, the O3 run concluded without another coincident detection of an EM counterpart. One reason is the larger distances at which the promising O3 candidates were reported or the fact that they had large sky-localization areas (see Coughlin (2020), for a summary).

The next few years brings a growing ground-based GW detector network that is reaching their design-sensitivity. There are $\mathcal{O}(10)$ BNS mergers expected in the fourth observing run (Abbott et al. 2018a) with a average range $\sim 190 \mathrm{Mpc}$. In the design sensitivity era this is expected to increase to $\sim 330 \mathrm{Mpc}$, which potentially implies a further $\sim 5$-fold increase in number of events detected. This increase in the number of events from GW facilities is matched by complementary advances in the optical and near-infrared, enabled by next generation telescope facilities like the Vera Rubin Observatory (Ivezic et al. 2019) ${ }^{3}$ and the Nancy Grace Roman Space Telescope (NGRST, Kasdin et al. 2020). Crucially for multi-messenger astrophysics, because these new facilities not only go deeper and cover a larger area on the sky at higher cadence than existing surveys, they have also catalyzed upgrades to existing facilities, making them more responsive. For example, telescope and observatory management systems (TOMS, Street et al. 2018) allow programmatic access to queue schedule a large array of facilities such as the Astrophysical Events Observatory Network (AEON, Street et al. 2020), increasing the likelihood of obtaining additional photometry and spectroscopy for GW sources within a few hours of explosions.

However, identifying the electromagnetic counterparts of BNS mergers in $\mathrm{O} 4$ and beyond presents a greater challenge to time-domain astronomy than run $\mathrm{O} 3$ since kilonovae evolve much faster than the typical cadence of these surveys. Discovering the counterpart requires that we are able to distinguish it from all of the other transient events also detected contemporaneously. The astronomical community has already wrestled with this challenge in O3 when several initially "interesting" candidates that were ruled out later, demonstrating the needle in a haystack nature of the problem. As a result, a targeted search for exotic transients like KNe will be polluted by contaminant "vanilla" objects, like supernovae. Followup time on large aperture facilities for these faint objects is extremely limited and it will not be possible to whittle down the list of potential counterparts by filtering out these contaminants using spectroscopy. While Integral Field Unit (IFU) spectroscopy is more promising in this respect, these facilities generally do not have large fields of view. Simply put, if EM followup in O3 was hard, followup in $\mathrm{O} 4$ is only going to get harder. Therefore, it is crucial to develop tools that will

3 The Vera Rubin Observatory was previously known as Large Synoptic Survey Telescope (LSST). Currently, LSST refers to the 10-year Legacy Survey of Space and Time that the observatory will conduct. filter interesting transients from the initial sparse photometry itself. This is the primary motivation for this work.

Given the large number of candidate counterparts from EM surveys, and the need for fast followup to monitor the rapid evolution of kilonovae, we focus on machine learning techniques to identify the counterpart photometrically. Several attempts at automated photometric classification of objects like supernovae have been reported earlier. Initial efforts relied on template fitting (Kessler et al. 2010a; Kessler et al. 2010b). Subsequent approaches utilized features extracted from such fits as features to train machine-learning (ML) algorithms (Narayan et al. 2018a). Lochner et al. (2016) utilized a non-parametric wavelet decomposition-based approach. The Photometric LSST Astronomical Time Series Classification Challenge (PLAsTiCC) (Kessler et al. 2019; Hlozek et al. 2020) was a global effort involving competing teams to classify $\mathcal{O}$ (millions) of simulated transients and variable lightcurves using machine learning (ML). All these attempts, however, only considered classification using the complete phase information of the lightcurve i.e. what the type of the object is, given that we have seen it rise, reach maximum light and fade away. These approaches are suitable to produce a complete catalog of photometrically-identified kilonovae retrospectively, and while this is important for studying the population of these objects, it does not enable real-time discovery and followup.

Photometric classification performance using only sparse initial photometry is considerably worse than performance when using the complete phase information. While early-time photometric information is necessarily sparse, and likely to be low $\mathrm{S} / \mathrm{N}$, wide-field optical and infrared surveys likely have access to considerable contextual information at the location of candidates. Classification of transients using only contextual information from their host galaxies, such as colors, light profile and redshift was first demonstrated by Foley \& Mandel (2013). Recently, Gagliano et al. (2021) further improved this approach considering an order of magnitude larger sample of the spectroscopically classified galaxies. Combining both early-time information and contextual information may provide a method to tackle the needle-in-a-haystack problem.

Of particular relevance to our work is the RAPID earlytime classifier described in Muthukrishna et al. (2019) (hereafter MU19). RAPID employs deep neural-networks (DNN) considering the early-time data as a time-series and mapping it to the transient class. The advantage is that the classification is provided with every epoch of data acquired. The computational cost is only encountered during training the DNN, with real time classification during nightly operations. The classifier can be further guided by providing contextual information. For example, in case of supernova classification, the knowledge about the redshift guides the classifier to give a better performance. Here, we leverage this feature - while the redshift may not be available for new objects from telescopes during the time of discovery of GW, there are several other data products that are provided during a GW discovery like the GW sky localization. Features of the skymap and its correlations with the objects discovered during follow-up, can serve a similar role and help in the filtering out the impurities which can save precious telescope resources. In this study, we modify the architecture used in RAPID, tuning it as a binary classifier for Electromagnetic Counterpart iden- 




Figure 1. An example scenario: the GW skymap associated with the $\mathrm{KN}$ is shown in the globe projection. The inset shows the sky locations of simulated transients. The $\mathrm{KN}$ is shown in the ' $\mathrm{x}$ ' symbol, the other objects are shown using circles.

tification (El-CID) i.e., to filter $\mathrm{KNe}$ from other transients during follow-up of GW candidates.

The organization of the paper is as follows. In Sec. 2 we describe the generation of our training data. We create an end-to-end training set starting with simulating BNS mergers in GWs. We generate skymaps for mergers that are detected by LIGO/Virgo. We then simulate KNe lightcurves associating the BNS mergers to KNe. We also generate other explosive transients which are supposed to be the contaminants during follow-up. In Sec. 3 we describe the training features and the contextual information that we provide during training. In Sec. 4, we talk about the neural network architecture and changes compared to RAPID. In Sec. 5, we evaluate the performance of the classifier on both simulated and real data. We find expected classification for real events like AT 2017gfo, counterpart of GW170817, and AT 2019npv, a supernova that was in the field of view of GW190814 and was initally considered of interest. We conclude in Sec. 6 highlighting the use case of a tool like El-CID for future follow-up operations.

\section{SIMULATING TRANSIENTS}

The situation we are considering is shown visually in Fig. 1 using a simulated GW sky-localization (skymap). The shading represents the probability density in the sky coordinates. If this is from a binary neutron star (BNS) merger, the associated $\mathrm{KNe}$ will likely lie in a region of high probability. We show the true location of the source with the $\times$ symbol. However, when attempting to identify the counterpart, we must contend with other unrelated transients that are temporally coincident and near the same high probability region
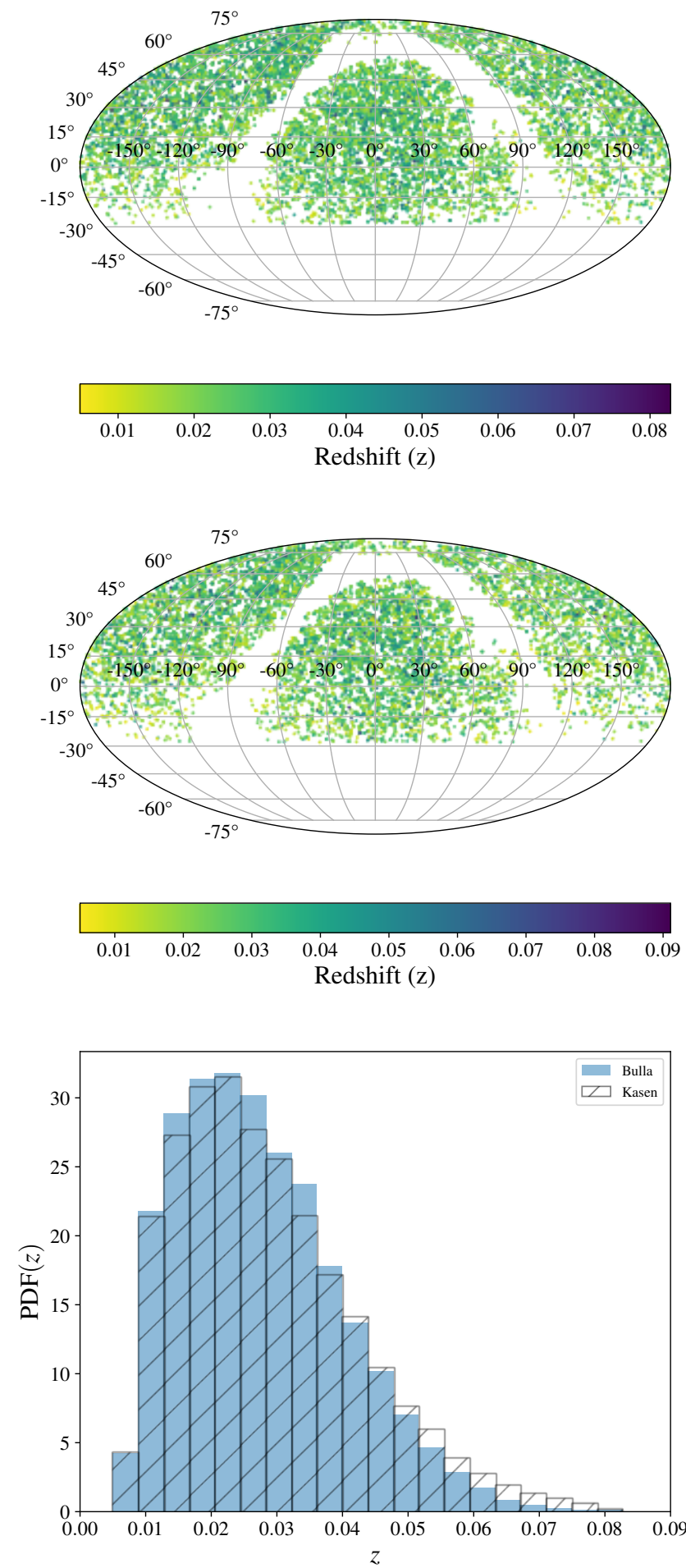

Figure 2. Upper panel: Distribution of the recovered Bulla $\mathrm{KNe}$ in the sky in Mollweide projection. The shading is based on the simulated redshift of the source. Middle panel: Same as above for the Kasen models. Lower panel: Distribution of the redshift for the recovered KNe for both models. The Bulla model case is shown in the filled histogram, while that for the Kasen model is shown in the hatched histogram. We observe that there is no preference towards detecting a specific model. 
defined in the GW alert. These events will also be discovered by surveys during their operations, and are shown by the solid circles. All these objects will have some photometric data; those with data before the GW trigger time are disqualified from being associated with the GW trigger. At the same time, if the first observation is made $\gtrsim 1$ week after the GW trigger, it is also likely unrelated since most KNe are expected to peak in optical bands $\sim 1-3$ days. Coincidental transients that satisfy the above criteria are the contaminants from which we must distinguish the kilonova.

During the initial hours through a few days after the GW alert, there will be a paucity of imaging data for all these sources for robust photometric classification. However, there is helpful contextual information that can guide a classification algorithm with the sparse data from the initial epochs of observation. Provided the LVC analysis pipeline is not biased, in general we can expect the true counterpart to be closer to the high-confidence region of the skymap, and the line-ofsight probability to the true event to be slightly higher than in random directions that are scattered about the true event. Given the location of the candidates, we can use the lineof-sight probability based on the skymap, and the angular offset from the mode of the skymap - as important contextual information to supplement the sparse initial photometry. While neither exclusively can provide a robust classification, in Sec. 5, we demonstrate that combining both using the neural network architecture in Sec. 4 does result in a reliable classification for the simulations we describe below, as well as GW170817 data.

To create an end-to-end realistic scenario like the one mentioned above, we generate a exhaustive set of simulated transients. This includes KNe and other explosive transients. We use the Supernova Analysis software package, SNANA (Kessler et al. 2009) for this purpose. While the scope of SNANA is broad, ranging from fitting lightcurves, classifying SNe, tools for SNe cosmology, and simulating explosive and variable transients, it is the last role that we are concerned with here. Given an empirical or spectral energy density (SED) model of an explosive transient along with the details of observing strategy and conditions of a survey i.e., its location, cadence, instrument thresholds, SNANA simulates lightcurves that would be observed by the survey including measurement errors. SNANA has been widely used in the literature for such simulations; a notable effort has been in generating $\sim 10^{6}$ of simulated transients and variable lightcurves for the Photometric LSST Astronomical Time Series Classification Challenge (PLAsTiCC) (Kessler et al. 2019). Other efforts regarding tools to simulate lightcurves include simsurvey (Feindt et al. 2019). Also, see Chatterjee et al. (2019) for a more rigorous treatment of detection efficiency of related to supernova rates.

\subsection{Simulating $\mathrm{KNe}$}

For our KNe, we use the spectral energy density (SED) model calculated on a kilonova model grid using the radiation transport code POSSIS (Bulla 2019, hereafter BU19), used earlier in Coughlin et al. (2020). We have incorporated this SED model grid in SNANA, and it can be accessed publicly as a

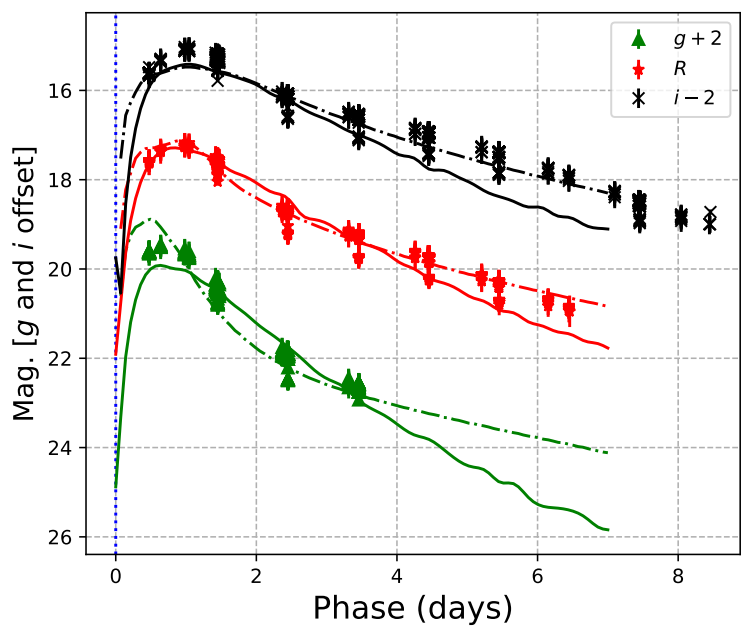

Figure 3. BU19 (solid curves) and KN17 (dotdash curves) models overplotted along with the DECam data from AT 2017gfo (offset for clarity). For the models in this figure, We use the best fit parameter model of the BU19 reported in Coughlin et al. (2020). For the KN17 model, we use one available publicly at https://github.com/dnkasen/Kasen_Kilonova_Models_2017; knova_d1_n50_m0.025_vk0.25_fd0.3_Xlan1e-6.0_vs0.32_ns12.0.h5

part of the SNANA package data. ${ }^{4}$ Each representative SED model is parameterized by the ejecta mass from the merger, $M_{\mathrm{ej}}$, the fraction of the lanthanide-rich component characterized by its half opening angle, $\Phi$, and the observing angle with respect to the line of sight, $\theta_{\mathrm{obs}}\left(\cos \theta_{\mathrm{obs}}=0\right.$ being edgeon, while $\cos \theta_{\text {obs }}=1$ being face-on). We also consider the KNe SED models by Kasen et al. (2017) (hereafter KN17), in which case the SED is parameterized by the ejecta mass, $M_{\mathrm{ej}}$, the lanthanide fraction, $X_{\mathrm{lan}}$, and the ejecta velocity, $v_{\mathrm{ej}}$. There is no observing angle dependence for the KN17 models. Given that there remains only one KNe definitively observed at electromagnetic wavelengths, there remain considerable model uncertainties, ultimately corresponding to different descriptions for the same physical system. In Fig. 3 we show example lightcurves from KN17 and BU19 models comparing them to AT 2017 gfo data from DECam. ${ }^{5}$ We simulate an equal number of events $\left(\sim 6 \times 10^{6}\right.$ each) using both KN17 and BU19 models, so as to not bias our machine learning algorithm towards either model. As the sample of observed kilonovae grows, we expect these different parametrizations to converge, and we can update the models as needed. We distribute the simulated events uniformly across the sky, and volume assuming a flat $\Lambda$ CDM cosmology with a Hubble constant, $H_{0}=70 \mathrm{~km} \mathrm{~s}^{-1} / \mathrm{Mpc}$, and matter density, $\Omega_{m}=0.3$. We consider an observing strategy based on ZTF's public "MSIP survey" observations included in the third data release of ZTF (ZTF DR3). ${ }^{6}$ While ZTF has had subsequent data releases, we are only concerned with those that over-

\footnotetext{
4 https://zenodo.org/record/4015340

5 Produced using some sncosmo wrapper code available at: https : //github.com/deepchatterjeeligo/bulla-models.

6 ZTF data releases includes observations from both "public" and "private" surveys. The public survey was the source of alerts sent
} 
lap with the LVC O3 run, so that our simulations reflect the published sensitives of both GW and EM surveys.

We simulate the sky brightness using magnitude limit data from ZTF DR3 and point spread function size distribution from Fig. 6 of Bellm et al. (2019). We evaluate the detection efficiency as a function of signal-to-noise using the distribution of the measured magnitude errors from the ZTF DR3 data release (see Appendix A for details). SNANA uses this information to determine the fraction of objects recovered at every epoch of detection. This process therefore includes realistic weather losses at the Palomar site. The distribution of the detected KNe in the sky is shown in Fig. 2 where the upper (middle) panel correspond to the BU19 (KN17) models. We find that the detectability peaks around a redshift of $\sim 0.025$, corresponding to a luminosity distance of $\sim 110$ Mpc.

\subsection{Simulating Other Transients}

For the several other transients that could be in the field of view, (see Fig. 1 for an example) we generate a representative sample of other possible objects. For this study we only use extra-galactic transients: SALT2 (Guy et al. 2007) type Ia supernovae (SNe Ia), core-collapse supernovae from the Nonnegative Matrix Factorization (SNII-NMF) model, (Kessler et al. 2019) more recent core-collapse supernovae by Vincenzi et al. (2019), (Vincenzi SNII), type Ibc supernovae, (SNe Ibc), type Iax (SNe Iax) supernovae, superluminous supernovae (SLSNe), and tidal disruption events (TDE). All these models are part of SNANA. These models, apart from the BU19 and the Vincenzi SNII model, were used in the PLAsTiCC challenge (see Sec. 4 and Fig. 1 from Kessler et al. (2019)). The difference, however, was the use of Rubin Observatory wide-fast-deep observing strategy in case of PLAsTiCC.

Here, we regenerate these with the same ZTF observing conditions library derived from ZTF DR3 and used for the $\mathrm{KNe}$ as described earlier. In Appendix B we show some example lightcurves from the training set. We do not make additional cuts on the dataset to balance the different types of objects. This choice is different from that used in MU19, as that worked focused on accurate multi-class classification, whereas our focus is on separating KNe from all other classes, which we regard as contaminants for the purpose of this work. While we use the BU19 and KN17 models and observing schedule of ZTF DR3 for demonstration here, the prescription can be easily applied to other lightcurve models, and future survey schedules, in particular Rubin Observatory and NGRST. The choice made here is motivated by the potential coincident survey operations with the upcoming fourth LIGO-Virgo-Kagra observing run.

The number count of the different types of objects are given in Table 1 . It should be noted that this is a simplification, and more accurately, the number counts depends on the relative rates of explosive transients, and their detection efficiency. While the relative rates of contaminating extragalactic classes may affect classification, we can expect some contaminants to be more difficult to distinguish than others
Table 1. The table lists the number count of different types of objects used for training

\begin{tabular}{cc}
\hline Object Type & Number count \\
\hline Bulla KN & 5126 \\
Kasen KN & 3707 \\
SALT2 SNIa & 24670 \\
SLSN & 16926 \\
SNII NMF & 24232 \\
SNIa 91bg & 28620 \\
SNIax & 21686 \\
SNIbc & 21258 \\
TDE & 19359 \\
Vincenzi SNII & 19909 \\
\hline
\end{tabular}

with only initial photometry. Here, we make a simple conservative approximation, and keep an order of magnitude more of the other objects compared to KN. This is motivated by our expectation that, while we are simulating events using ZTF's untargeted survey strategy, most efforts using the tool developed in this work will be applying it to images acquired through a dedicated targeted search, precisely because they are trying to identify the EM counterpart immediately after receiving a GW alert. While the $\mathrm{KNe}$ volumetric rate is roughly one-thousandth the local SN Ia rate, given the context of a target-of-opportunity search for $\mathrm{KNe}$, the number of $\mathrm{KNe}$ discovered will inevitably be disproportional to their volumetric rate. We have verified that the extreme case of repeating training with a roughly equal number of objects instead - modeling a target-of-opportunity search that is unreasonably effective - gives a similar result. In Sec. 3 (see Table 2), we further repeat the training/validation process for different $\mathrm{KN} /$ non-KN object proportion and do not observe significant difference in performance. The other extreme - a machine learning algorithm conditioned on a training set that includes objects in proportion to their volumetric rate and detection efficiency - is unsuitable for our goal of detecting kilonovae, but may be of use to groups looking to retrospectively classify all objects from a survey. It such a case if an imbalance is necessary for technical/practical reasons, the output needs to be re-weighted based on rates and detection efficiency of different object types.

\subsection{Simulating Sky-localizations}

The GW alerts contain information about the merger time, how many ground-based interferometers detected the signal, and the significance of the candidate. They also contain additional data products that aid the hunt for any potential EM counterpart. ${ }^{7}$ For follow-up purposes, the sky-localization is of particular importance since it determines scheduling and observing strategy. Additionally, the possibility of encountering contaminants increases drastically with increasing skylocalization area. We compute and associate a GW skymap with each KNe lightcurve instance described in the previous section.

The basic principle of sky-localization utilizes the difference

\footnotetext{
7 https://emfollow.docs.ligo.org/userguide/content.html
}

to brokers and was used to build a survey with predictable average cadence. 


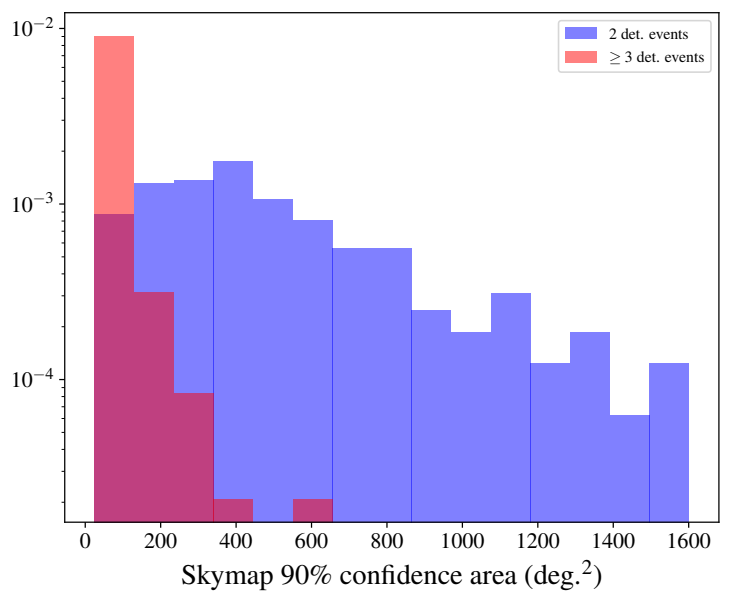

Figure 4. Distribution of the $90 \%$ confidence regions of the 2detector vs $\geq 3$-detector events in the training set.

in the time of arrival of the signal between the GW detectornetwork to localize the source. The GW signal depends on the intrinsic properties of the binary, like the masses, and spins of the binaries, and also extrinsic parameters like the distance and inclination with respect to the line-of-sight. The detectability of the signal, and the measurement uncertainties, like the localization area in sky, depends on the signalto-noise ratio $(\mathrm{S} / \mathrm{N})$ produced at the detector. On the other hand, the amount of ejecta released from the aftermath of NS merger depends on the complex high-energy astrophysics of the merger environment, and the NS equation of state (EoS) (for example, see Shibata \& Hotokezaka 2019, for a review). While the accurate answer to ejecta properties is given by numerical relativity (NR) simulations, empirical fits to NR results have been performed to estimate the ejecta mass given the binary source parameters and the NS EoS. Here, we use such a relation due to Dietrich \& Ujevic (2017) (hereafter DU17) as implemented in the gwemlightcurves library ${ }^{8}$ used in Coughlin et al. (2020). The DU17 relation provides the ejecta mass given the binary parameters as,

$M_{\mathrm{ej}}=M_{\mathrm{ej}}\left(m_{1,2}, C_{1,2}\right)$,

where $m_{1,2}$ are the component masses and $C_{1,2}=$ $\left(G / c^{2}\right)\left(m_{1,2} / R_{1,2}\right)$ is the compactness of each component. The compactness is a function of the equation of state (EoS) of NSs. While there is still uncertainty in the true NS EoS, the tidal deformability measurement from GW170817 data, (Abbott et al. 2018b) and the constraints on mass and radius from NICER (Miller et al. 2019) have ruled out several EoSs in literature. Recently, Ghosh et al. (2021) have performed model selection of the EoSs in the literature against GW170817 data. For this study, we make a choice of the APR4_EPP EoS, which is well motivated physically, and well supported by GW170817 data. This is justified because the ejecta mass is not a strong function of the compactness if we restrict to the set of EoS that have support from mass-radius measurements from GW170817 or NICER. We show this in

\footnotetext{
8 https://gwemlightcurves.github.io/
}

\begin{tabular}{cc}
\hline \hline Fraction of non-KN objects & AUC at 3rd day after trigger \\
\hline Complete set & 0.99970 \\
0.50x non-KN objects & 0.99957 \\
0.33x non-KN objects & 0.99950 \\
$0.25 \mathrm{x}$ non-KN objects & 0.99881 \\
\hline
\end{tabular}

Table 2. Area under the (receiver operator statistic) curve (AUC) at 3rd day after trigger. We progressively reduce the fraction of non-KN objects in the training set using a fraction of the total non-KN objects in the total set each time to test the impact of relative imbalance of objects towards binary classification. We observe that the relative imbalance does not affect the binary classification performance significantly.

Appendix C. For each KN lightcurve, parameterized by $M_{\mathrm{ej}}$, we solve the DU17 relation to obtain the component masses of the binary. We ignore spins for this analysis. Note that the solution from $M_{\mathrm{ej}} \rightarrow m_{1,2}$ is not one-to-one. In practice, we obtain the solution using a stochastic technique - we sample uniformly in chirp mass and mass ratio, obtain the component masses, and use Eq. (1) to match the ejecta mass value to the nearest BU19 SED $M_{\text {ej }}$ grid-point. We also use the $\theta_{\text {obs }}$ parameter as the inclination of the binary orbit with respect to the line of sight. For the KN17 models, we follow the identical procedure to obtain binary parameters from the ejecta mass, but associate a random inclination angle based on a uniform prior on cosine of the inclination. In this manner we obtain the binary parameters.

We then use the binary parameters to generate GW strain using an analytic waveform approximant, (Arun et al. 2005) ${ }^{9}$ and compute the signal-to-noise $(\mathrm{S} / \mathrm{N})$ time series in the HLVK detectors based on different noise realizations. We consider the projected sensitivity in the LIGO/Virgo/KAGRA fourth observing run $(\mathrm{O} 4)$ and the design-sensitivity expected in the fifth observing run (O5). To generate the $\mathrm{S} / \mathrm{N}$ time series, we use the bayestar-realize-coincs tool from the ligo.skymap library. ${ }^{10}$ This simulates a match-filter search using gaussian-noise. We impose a threshold of $\mathrm{S} / \mathrm{N} \geq 4$ for a single GW detector, a network $\mathrm{S} / \mathrm{N} \geq 12$, and a coincidence in at least two detectors to claim discovery in GWs. Subsequently, we run the low-latency sky-localization code BAYESTAR (Singer \& Price 2016) on the detected sources to obtain a skymap associated with each KN lightcurve. In Fig. 4 we show a comparison between the $90 \%$ skyareas of the two-detector and greater than two-detector events.

\section{TRAINING DATA}

\subsection{Training set}

With each simulated kilonova light curve associated with a GW trigger alert and skymap, as well as the simulated light curves of contaminants from multiple plausible astrophysical classes, we preprocess the events and label events to assemble the training set as follows:

9 TaylorF2 correct up to 3.5 post-newtonian order in amplitude and phase

10 https://lscsoft.docs.ligo.org/ligo.skymap/ 


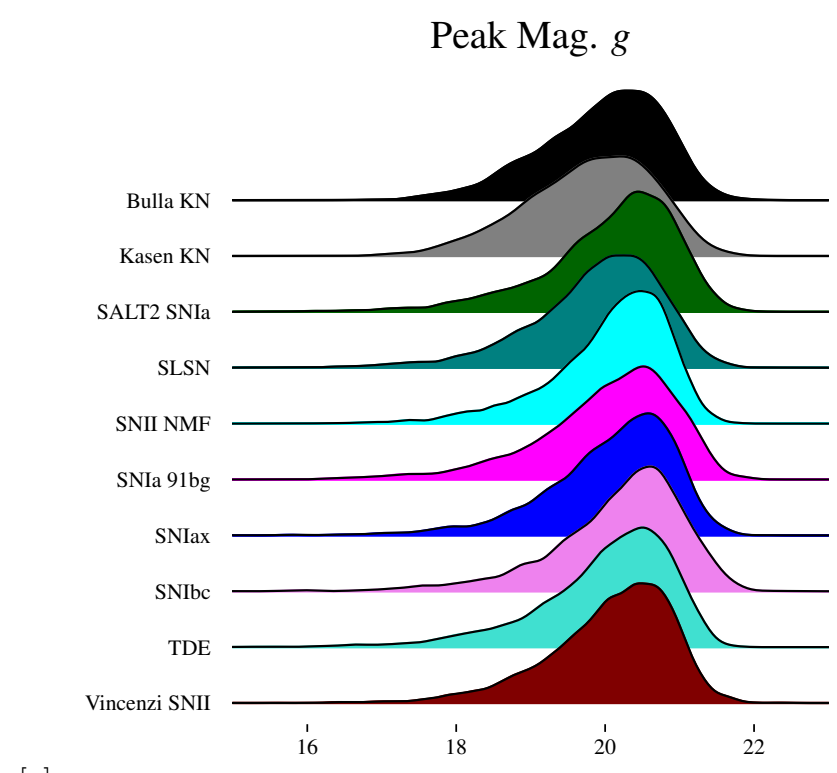

[p]

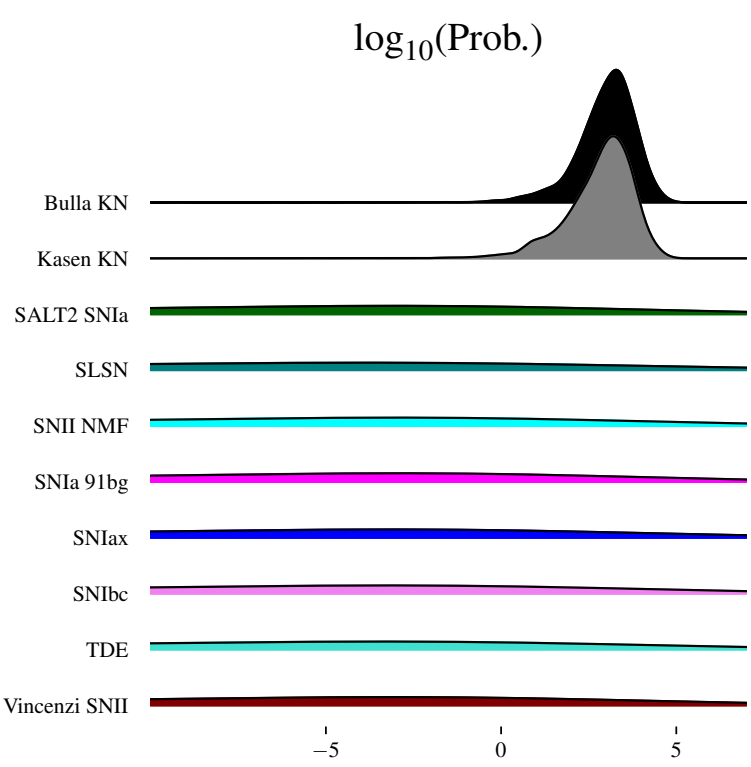

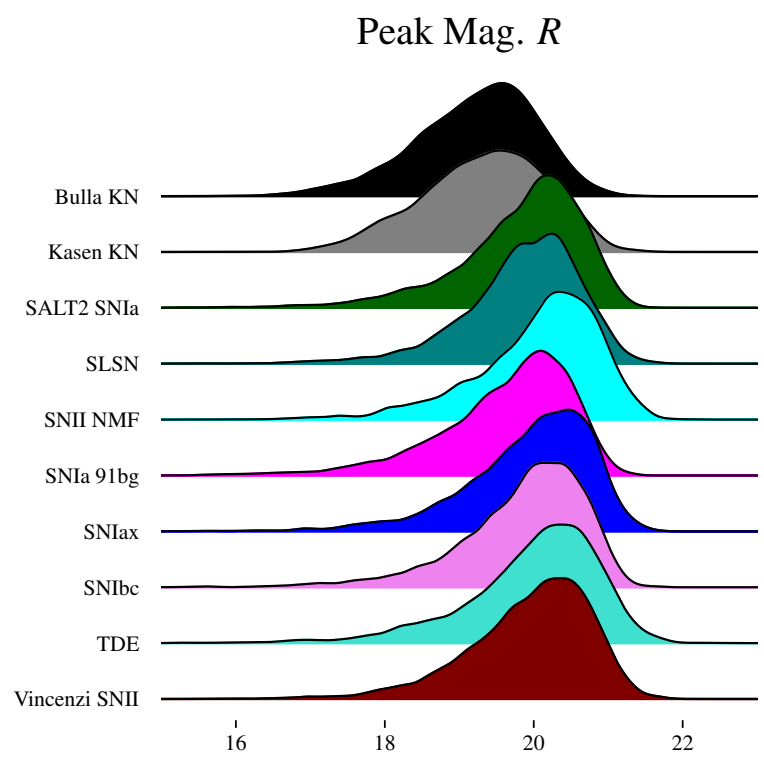

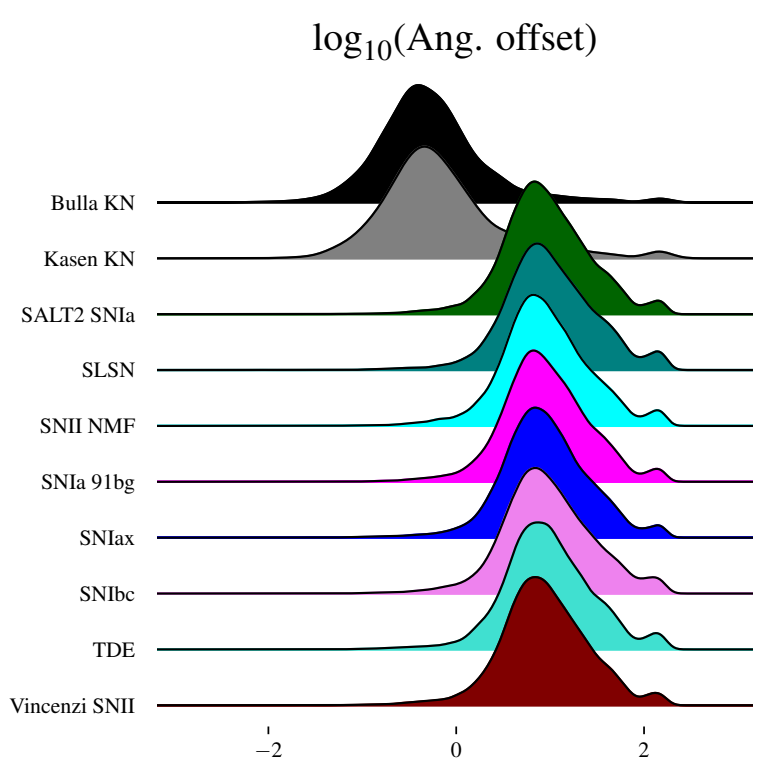

Figure 5. Upper panel: Peak $g$ and $R$ magnitude distribution. As expected, there is no preference for a particular object type in terms of the observed flux. Lower panel: Distribution of line-of-sight probabilities and angular offsets in degrees from the mode of the skymap

- We assume the explosion time of the kilonova to coincide with the GW trigger. This is justified since the time to blackhole formation from the merger of neutron stars is $\mathcal{O}(10-100)$ (milliseconds; see Radice et al. (2018) for example), at which point the dynamical ejecta is expelled.

- For each kilonova in our sample, we consider any contaminant light curve in our simulated sample that has a first detection within 7 days of the GW trigger. With the dense set of simulations, this step yields $\mathcal{O}(100-1000)$ objects, most of which have high angular separation from the high probability density region in the GW skymap corresponding to the kilonova.

- We rank these objects based on their line-of-sight probability, and select the top 20 objects for each KN/skymap combination. Thus, our training set contains about $20 \mathrm{x}$ the num- ber of KN lightcurves we start out with. For this study we use about $8 \times 10^{3} \mathrm{KN} /$ skymaps combinations, which implies that our training set has $\sim 1.6 \times 10^{5}$ total lightcurves/contextual information combinations. We have verified that the performance is not affected by changing the proportion of KN vs. non-KN objects in the training set. In Table 2, we show the area under the receiver operator statistic curve on the 3rd day after trigger by repeating the training/validation changing the proportion of KN vs non-KN objects each time. We do not observe a significant difference in performance.

- The KN (BU19 or KN17) are labeled as "Kilonova" while the other objects are labeled as "Other" class.

In the upper panel of Fig. 5, we show the distributions of the $g$ and $R$ observed band fluxes as output by SNANA. As expected, since all the events are simulated using the same 


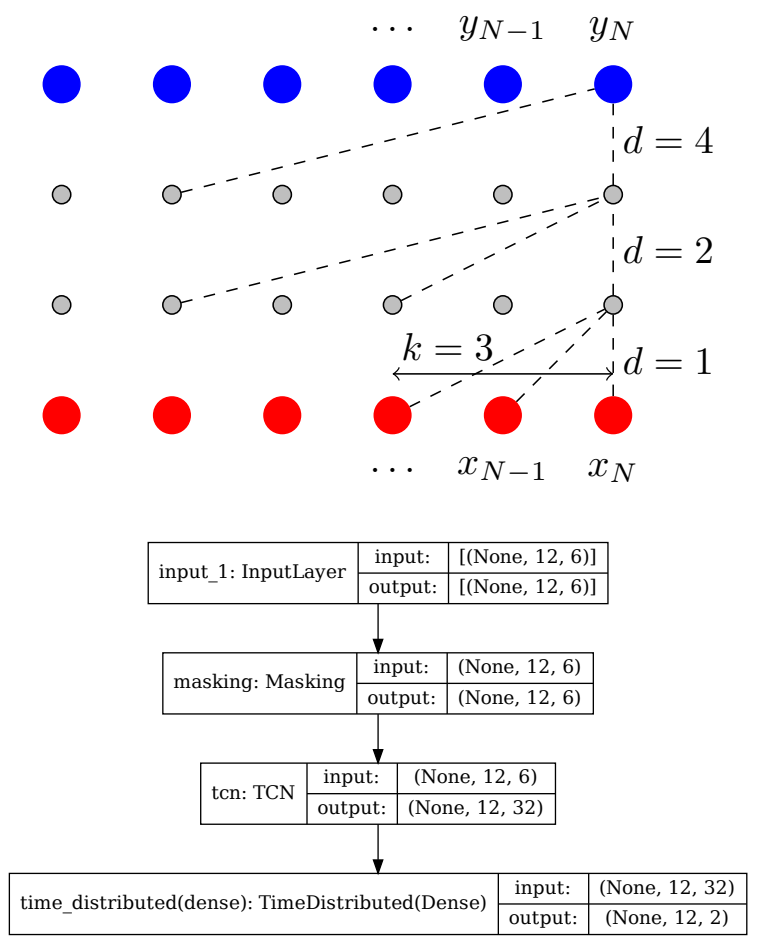

Figure 6. Upper panel: A basic architecture of a TCN, reproduced based on Fig 1. of Bai et al. (2018) (see for more details). The sketch shows the causal nature of the convolutions - output $y_{N}$ depends on the input till $x_{N}$. The filter length, $k$, and the dilations, $d$, control the preservation of history of the time-series. Lower panel: Layers implemented using keras, showing input and output shape at each step.

survey properties, there is no significant bias that can discriminate any specific object type. While the distribution of peak absolute magnitudes and colors can differ between the classes, the observed magnitude distributions are largely similar as they are determined by the survey capabilities. With only sparse early-time photometric data, it would not be possible to discriminate between kilonovae and other classes effectively. However, in the bottom panel we show the distribution of the line-of-sight probability and the angular offset from the mode of the GW skymap for all the objects. These distributions sets the KNs apart from the other classes, as the GW skymap is associated with the kilonovae, whereas any association between the skymap and a light curve from some other class of object will arise purely by coincidence. When considering a population of contaminating events, no correlation will persist between the GW skymaps and the light curves. This highlights the value of considering contextual information in addition to the light curve, particularly for early classification, even if the contextual information is drawn from a different survey.

\section{BINARY CLASSIFICATION}

We use a similar neural network architecture as RAPID. While the original architecture used gated recurrent units (GRUs), we use a temporal convolutional network (TCN) ar- chitecture (Bai et al. 2018) as implemented in keras (Remy 2020). ${ }^{11}$ As mentioned earlier, here we are only concerned whether an object is/is not a KN. Thus, we modify the architecture used in MU19 to a binary classifier to act as a filter for $\mathrm{KNe}^{12}$

\subsection{Temporal Convolutional Network}

A TCN architecture Bai et al. (2018) uses two basic principles - 1) the output is of the same length as the input, and 2) the internal convolutions are causal. To illustrate this point, consider a sequence, $\left\{x_{1}, \ldots, x_{N}\right\}$; the output is a sequence $\left\{y_{1}, \ldots, y_{N}\right\}$, and the convolutions of the hidden layers of the architecture are such that the output, $y_{n}$ only depends on $\left\{x_{1}, \ldots, x_{n}\right\}$, where $n \leq N$. We show a basic sketch in Fig. 6 . While TCNs have previously been used in the literature to model periodic variable stars, (see Martínez-Palomera et al. 2020 , for example) this is the first use of this architecture to classify astrophysical transients.

There are two ways to preserve temporal long-term history of the input sequence: using larger filter ${ }^{13}$ length, and using dilated layers of causal convolutions in this architecture. Increasing both correspond to extraction of features that are correlated farther in time. In our case, the temporal data is the flux of the object as a function of time i.e., the lightcurve. Additionally, the contextual information is also an input, but its value does not change with time, for example, the angular offset of the object from the mode of the skymap, or its line-of-sight probability. Following MU19, we sample the lightcurve uniformly in $N$ steps. Our input matrix, therefore, has dimensions of $N \times(p+k)$, where $p$ is the number of passbands ( 3 for ZTF), and $k$ is the number of contextual information features. In this case, this includes the line-ofsight probability, the angular offset, and the $90 \%$ area of the skymap.

The light curves are preprocessed before training. The flux for any object is approximated using linear interpolation in between two observations, and then sampled at regular intervals to get the input time-series, $\left\{x_{1}, \ldots, x_{N}\right\}$. In order to find the regular intervals, we consider the 80 percentile value of $\sim 0.7$ days as the time difference between two successive observations of our simulated light curve. While this is an empirical choice and can be changed, for the cadence of observations used by ZTF, using a much larger interval decimates the time variability, while using a shorter interval causes jitter due to interpolating in an interval with sparse data. We only consider the light curve flux within 10 days from the GW trigger for all simulated object. This limit arises from the physics of kilonovae itself. Even the most intrinsically bright kilonovae evolve over $\sim 1$ week after the BNS merger. If an EM counterpart cannot be identified within 10 days, we simply flag the event as failed, as kilonovae become too faint at optical wavelengths for any follow-up studies that begin after 10 days. This choice differs from that used in MU19, which focused on multi-class classification rather than kilonova counterpart identification. Within this 10-day window,

11 https://github.com/philipperemy/keras-tcn

12 At the time of publication, this is a separate branch of the RAPID codebase.

13 called "kernel" in the keras nomenclature 

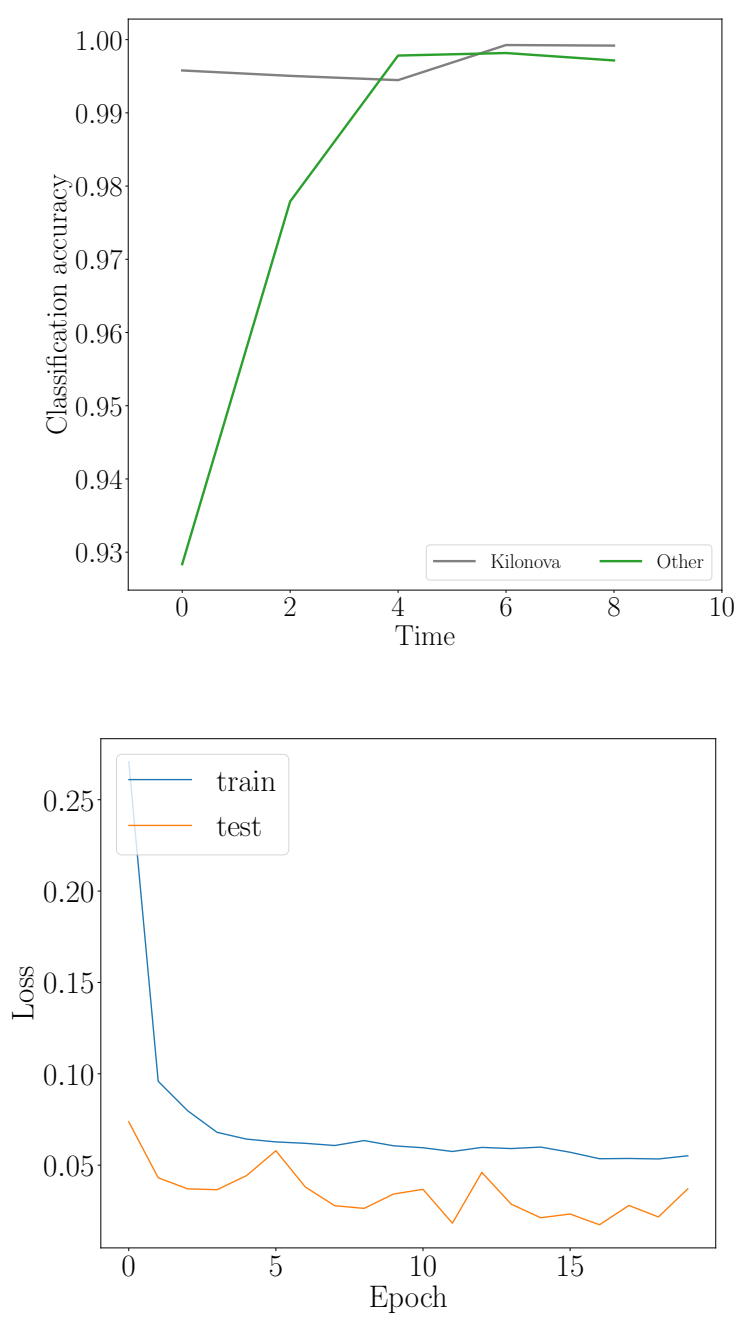

Figure 7. Upper panel: The accuracy of prediction with time for the binary classifier. Lower panel: The loss as a function of the training epoch.

we interpolate the flux linearly onto an evenly spaced $N=12$ grid. This choice satisfies $0.7 \times 12 \gtrsim 1$ week, giving us sufficient time resolution to incorporate observations from most time-domain surveys with cadences of $\gtrsim 1$ day, while also covering the KN evolution. Thus, our input matrix has a shape of $12 \times 6$. A summary of the dimensions of the input, output, and hidden layers in the bottom panel of Fig. 6 .

\subsection{Training}

The kernel size of the hidden layers control the number of trainable parameters in the network. In addition, from going from one hidden layer to the next, the input from the previous layer is passed through a residual block (He et al. 2015). This is done to avoid vanishing gradients during training. In this step the input from the previous hidden layer is passed through two causal convolutional layers and a dropout before being added to itself. This further increases the number of trainable parameters. For our network, we use a filter length, $k=2$, and dilation layers, $d=\{1,2\}$. We use a kernel size of 32 for the hidden layers (not shown in Fig. 6). We also use 2 stacks ${ }^{14}$ to increase the depth of the network. The total number of trainable parameters for the network is $\sim 13000$. We use a dropout rate of $5 \%$ to prevent overfitting. We use the categorical cross-entropy as the loss function, and the Adam optimizer. We find that $\sim 20$ epochs of training $(\sim 5$ minutes total on 8 Intel core-i7 processors) is sufficient to minimize the loss. We use $60 / 40 \%$ of the data for training/validation fraction for our dataset. In Fig. 7, we show the accuracy per class with time, and loss as a function of the training epoch. It should be observed that for most of the $\mathrm{KNe}$, their sparse photometric data implies that the class is estimated solely from their contextual information i.e., consistency with the GW skymap. For the other objects, however, the photometric information is responsible for classifying them correctly i.e., we see the accuracy increase with time. In either case, the $\geq 99 \%$ accuracy is reached after $\sim 3$ days from trigger.

We would like to mention that the end-to-end dataset preparation i.e., simulating binaries, running BAYESTAR to create skymaps, and using SNANA to prepare realistic lightcurves, is the computationally expensive step in this work. We find that the computational cost in training the neural network is significantly less, not requiring hardware optimizations, like the use of GPUs, at this stage. We attribute this to the short duration photometry i.e., the input arrays are relatively small compared to that needed for supernova lightcurve classification as in MU19. Also, we only use certain features of the skymap to capture the correlations between skymap and the location of other objects, instead of using the complete HEALPix map as a feature vector. However, certain hardware optimizations, like GPU support, may be done for future scalability since the underlying infrastructure of tensorflow natively supports GPUs. In the next section, we further validate the performance on simulated data that was not included in training, as well as the real observations of GW170817, through similar passbands, but at different facilities than our simulated ZTF training set.

\section{RESULTS ON UNSEEN DATA}

\subsection{Performance on GW170817}

The most natural choice to test the performance of El-CID is AT 2017gfo, the kilonova from GW170817. We use the publicly available data from the Open Kilonova Catalog. ${ }^{15}$ This dataset is a compilation of the photometry from the various instruments that participated in the follow-up of AT 2017gfo, and was not a part of our training sample. In Fig. 8, we show the data for the first few days after the trigger for a subset of instruments with optical passbands, similar to what we have used. We do not perform any $k$-corrections to the magnitudes. We only consider the observation in filters used for training $-g, R, i$. Therefore, the data is extremely heterogeneous involving different cadence and instrument properties.

Upon passing this to El-CID, we find that KN class is weighted strongly at the first detection, which is expected since AT 2017gfo lied in a high confidence region of the skylocalization. We find that irrespective of the instrument, the

14 nb_stacks=2 in keras-tcn.
15 https://kilonova.space/ 

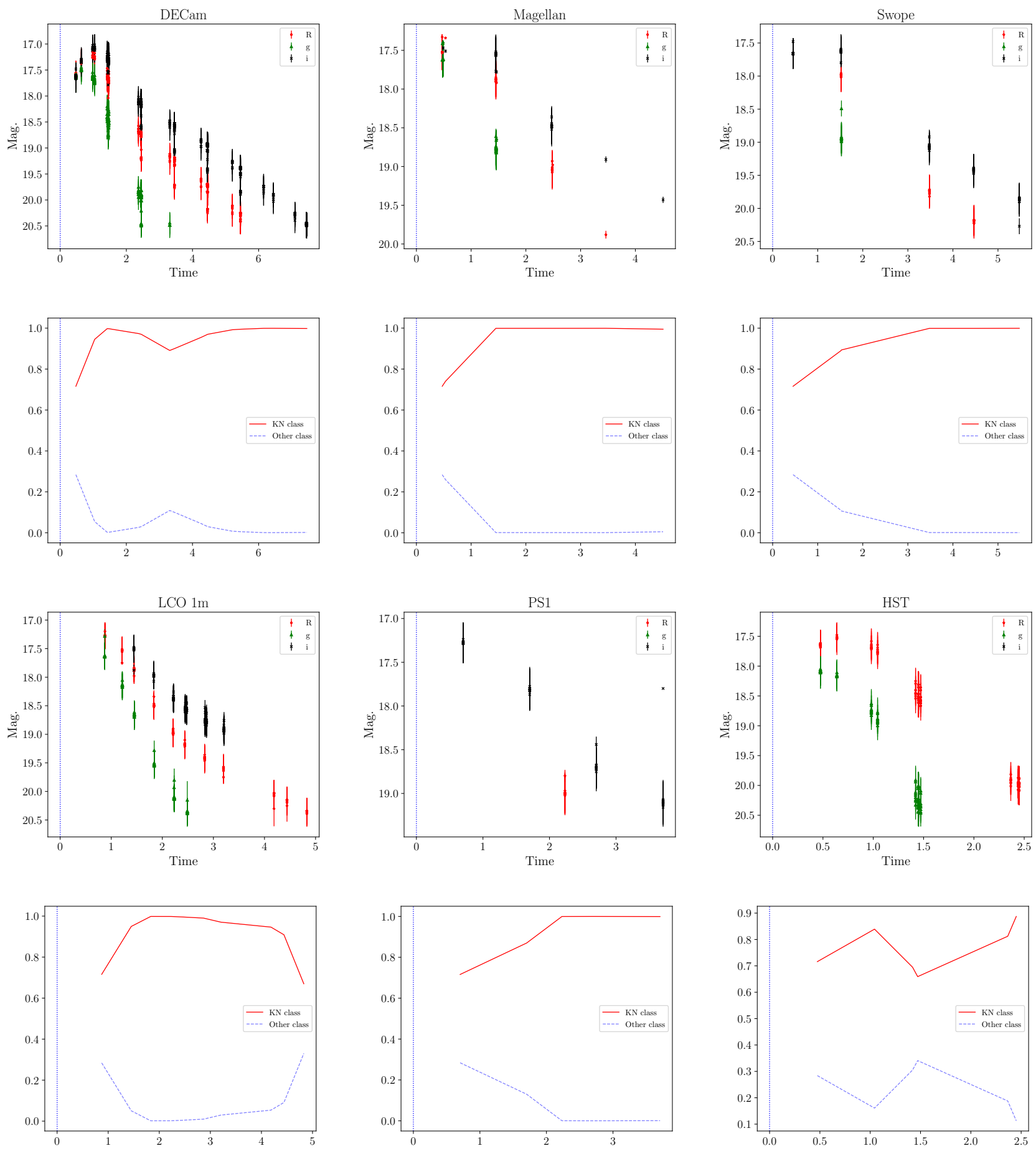

Figure 8. Predictions of the classifier on $\sim 1$ week data for GW170817 obtained from the Open Kilonova catalog.

classification stays correct. This shows that the classifier is robust to cadence and the temporal features are picked out instead.

In some cases, like the LCO- $1 \mathrm{~m}$ case, the there is a decrease in KN class score at larger time intervals - this is a known feature. While most of the KNe in the training sample have observations in under a week from trigger, the LCO$1 \mathrm{~m}$ lightcurves stay above threshold beyond a week as they were observed with exceptionally long exposures. These light curves therefore show a tendency of a drop in $\mathrm{KN}$ score in the later epochs as the real long-exposure observations are not similar to our training data which use a constant exposure as would be employed by an untargeted search. A possible solution to be considered in the future is the augmentation of the training set by including simulations with either a mix of small and large aperture telescopes, or by incorporating short and long duration exposures.

In Appendix D, we also show the results from a classifier 
trained on $\geq 3$-detector coincidences in the design sensitivity era. We find that the results are not significantly affected. This is expected because the detection of the joint sources are mostly limited by the sensitivity of the telescope for future observing eras.

\subsection{Performance on AT 2019npv}

Next, we consider the performance on a type Ibc supernova, AT 2019npv, that was found in a region of high-confidence in skymap of GW190814 (Abbott et al. 2020b). We show the skymap and the location of the transient in Fig. 9. GW190814 was the best localized event during LVC run O3, with a preliminary skymap localization area of $\sim 20$ sq. degrees. This event was interesting for follow-up since it was reported to have strong chance of having a NS component (mass less than $3 M_{\odot}$ (LIGO Scientific Collaboration, Virgo Collaboration 2019). Several teams across the globe participated in the follow-up effort of this event (Antier et al. 2019; Dobie et al. 2019; Andreoni et al. 2020; Morgan et al. 2020; Vieira et al. 2020; Watson et al. 2020; de Wet et al. 2021). AT 2019npv was discovered by Goldstein et al. (2019) and confirmed by several groups (Herner et al. 2019; Smartt et al. 2019; T.W. Chen et al. 2019; V. Lipunov et al. 2019; X. Wang et al. 2019). The redshift of this source was consistent with the distance information from the skymap. We show the observations taken from Andreoni et al. (2020) in the middle panel of Fig. 9. The initial $i$-band observations in the first week were taken with DECam. The later observations are from a mix of several instruments at different facilities. The data is taken from Fig. 3 of Andreoni et al. (2020).

We find that as expected, the initial KN score is high due to the consistency between the event location with the highconfidence region of the GW skymap. However, every subsequent epoch of observation drives the KN score down as El-CID sees more of the evolution of the light curve, the contextual information carries less weight and the algorithm becomes more certain that this event is not a kilonova.

This serves as another verification of the classifier performance on unseen real data. In Appendix D, we also show the results from using a training set in the design sensitivity era.

\subsection{Performance on mock KN lightcurves in Rubin Observatory Era}

Finally, to verify the performance on a larger sample, and validate the insensitivity towards cadence, we test the performance of the mock KN simulated using the Rubin Observatory "minion_1016" cadence for the wide-fast-deep (WFD) survey. This was the previous baseline cadence adopted by LSST, and was used in PLAsTiCC (Kessler et al. 2019; Hlozek et al. 2020). We repeat the steps in Sec. 3 using the BU19 models to check the performance. It should be noted that the training set does not contain these simulations. We still use the $\mathrm{O} 4$ noise PSD to generate the skymaps. Therefore, our test models a hypothetical scenario where Rubin Observatory is in operation during the $\mathrm{O} 4$.

We consider simulations that are within $\sim 100 \mathrm{Mpc}$ distance. We consider only the part of the lightcurves where the recovered magnitudes are consistent with ZTF's threshold i.e., brighter than 20.5. This results in lightcurves that
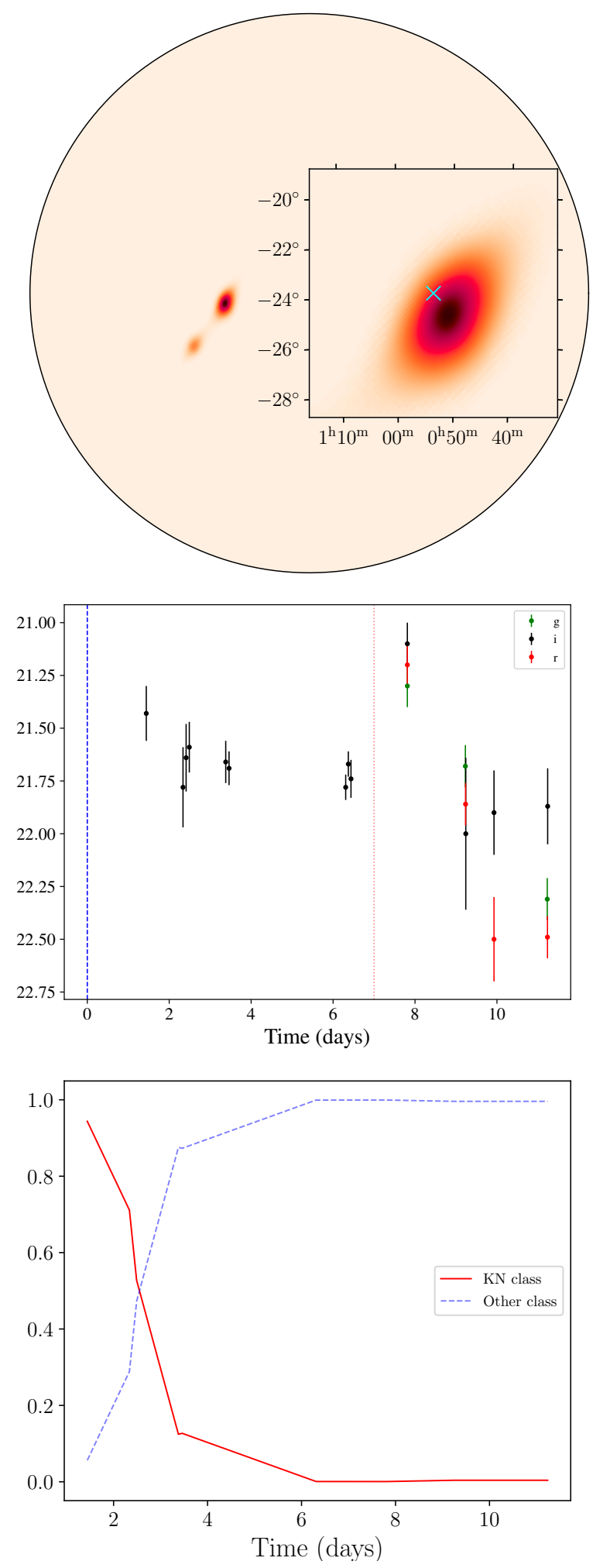

Figure 9. Upper panel: The skymap of the GW190814 event. The cross shows the position of AT 2019npv, a SN Ibc. The object is in a high-confidence region of the skymap, and was considered a KN doppleganger few days into discovery (Andreoni et al. 2020). Middle panel: The photometry of AT 2019npv taken from Andreoni et al. (2020). The reference time is the merger time of GW190814. The observation till $\sim 7$ days (dotted vertical line) is from DECam follow-up. Subsequent observations are from several observatories. Lower panel: El-CID results on AT 2019npv. We find, as expected, the initial contextual information weights the

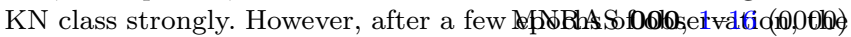
KN prediction drops. 

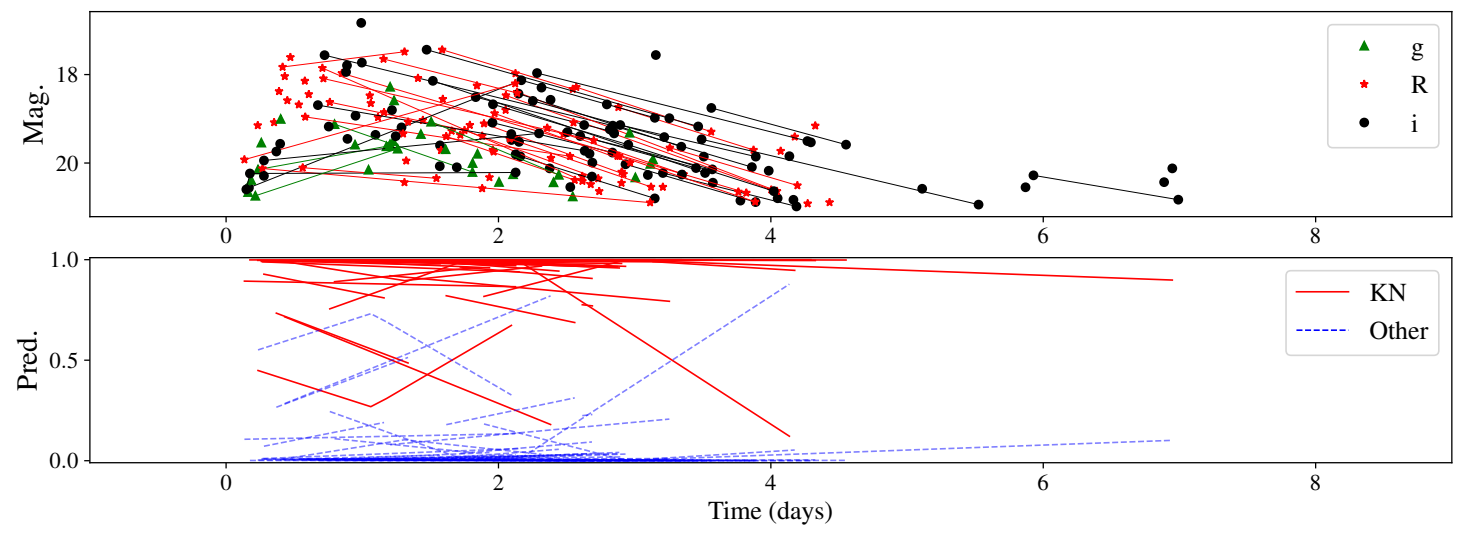

Figure 10. Upper panel: Simulated KNe lightcurves using the Rubin Observatory WFD survey cadence. These were not a part of the training sample for El-CID. We also generated skymaps for these events using the procedure outlined in Sec. 2. Lower panel: El-CID predictions on the unseen lightcurves. Most of the lightcurves are correctly classified. This is a verification that the classifier is learning the temporal features and not the cadence of the training set.

are around a week long. As mentioned above, due to the lack of the training set containing KNe that are observed for larger timescales, the classifier suffers from the caveat that lightcurves longer than $\sim 1$ week have a tendency to be misclassified.

In future work, we plan to make the classifier more robust to instrument thresholds by including simulations carried out with different instruments. We overlay $\sim 100$ of these lightcurves in the top panel of Fig. 10. In the lower panel, we show the performance of the classifier on these and find that most of the objects are correctly classified - only $2 / 115$ have their final classification score as the "Other" class, which further validates the performance on unseen data.

\section{SUMMARY AND CONCLUSION}

The third LIGO/Virgo observing run dramatically illustrated the challenge faced by multi-messenger astrophysics - even as more GW events are identified, locating their EM counterparts quickly becomes daunting because of the large area that needs to be searched, and the large number of contaminating transients that must be filtered out automatically. Additionally, most kilonovae are not expected to be like GW170817/AT 2017gfo, and locating these faint counterparts before they fade into irrelevance demands novel techniques. Here, we have considered a machine learning approach that combines the sparse low $\mathrm{S} / \mathrm{N}$ optical observations together with contextual information that is already provided with the GW alert, to increase the odds of being able to identify the counterpart. Our results reinforce the utility of deep neural networks in astrophysics, to emulate tasks that previously required visual inspection of the data by expert observers. These techniques will become ever more essential as surveys such as the Rubin Obsevatory discover more sources than can ever be inspected by humans.

In this article, we have re-purposed the RAPID neural network architecture into El-CID, tuned for filtering KNe from contaminants. The former has already been shown to produce promising results, and is used classifying real-time alerts from ZTF as a part of the ANTARES ${ }^{16}$ broker (Narayan et al. 2018b; Matheson et al. 2021). While the major challenge towards classifying fast transients like kilonovae is the sparse data, the use of the other contextual information available during discovery time has not been made use of in a systematic way i.e., built into a classification algorithm. This work developed the infrastructure to create realistic end-toend GW binary merger simulations, creating a skymap for each, and simulating an associated KN lightcurve in a synoptic telescope like ZTF. The procedure we outline allows us to create a rich dataset which emulates the correlations between the photometric and GW observables. We also simulate the sky - other objects that can be contaminants during the search for a counterpart in a GW follow-up. We then supply both the photometric and the contextual information like the line of sight probability, the angular offset from the mode of the skymap, and the $90 \%$ confidence sky-localization area as training feature to help the classifier differentiate between objects even in the absence of good photometric data. We note that while Stachie et al. (2020) have used the model trained in MU19 towards classifying KNe, the use of GW discovery data products and its correlations with photometric information has not been done earlier to our knowledge. Also, the MU19 model is directed toward SNe classification. For example, in the pre-processing step of MU19, the lightcurves are interpolated at a 3-day interval, which is sufficient to capture the temporal features of a $\mathrm{SNe}$, but is large compared to that of a typical KNe. Apart from using a training set more dense in $\mathrm{KNe}$, we have also adjusted the neural network to shorter timescales. Although we have restricted to other extra-galactic explosions as contaminants in this work, the extension to add more contaminant object types, like galactic transients, is readily done using SNANA which is optimized for high throughput. For example, in this work the runtime for simulating $\sim 6$ million $\mathrm{KN}$ simulations with single node and $20 \mathrm{CPU}$ cores was $\sim 7$ hours. We, therefore,

16 https://antares.noirlab.edu 
expect the procedure to scale in terms of adding other object types. An interesting fast galactic object type in this regard are M-dwarf flares occurring in the galactic halo. Archival searches for fast optical transients have led to discovery of several such objects (for example, see Ho et al. 2018). In such a case, however, other contextual information like the offset from the galactic plane may be used as another feature during training. This will be considered in the future. While complete photometric classification into different classes has already been achieved with RAPID, the key problem we will face during LVK $\mathrm{O} 4$ and beyond is to filter out the $\mathrm{KN}$ from the rest of the alert stream from wide-field surveys. We therefore framed El-CID as a binary classifier, and show that the use of temporal convolutional networks makes the network largely independent of the observing cadence. We achieve a $\sim 99 \%$ accuracy with our training sample, and also verify the performance of the classifier on unseen data. We find that the classifier works expectedly for the only EMGW event, GW170817/AT 2017gfo, and the classification is correct across the data taken by different instruments. We also analyze the case of AT 2019npv which was a contaminant in the follow-up effort of GW190814, and find that the classifier is able to rule out the object in the first few epochs of observation.

Tools like El-CID will be crucial in the follow-up efforts in the future. We would like to stress that the use case is for broker teams to slice through the candidate list in near real time after discovery. We would also like to point out that the values given by El-CID is to be interpreted as a score, and is not analogous to an evidence from a bayesian inference. Retrospective analysis on a population should use the values from El-CID accordingly. We expect the El-CID architecture can be periodically trained from nightly data taken from public brokers like ANTARES during Rubin Observatory operations. Dedicated public EMGW efforts like the Scalable Cyberinfrastructure for Multi-messenger Astrophysics (SCiMMA) ${ }^{17}$ could have El-CID running as an annotation service. The results provided by El-CID could be used by downstream facilities to schedule observations. Recently, there have been conceptually similar efforts like the ZTFRest framework (Andreoni et al. 2021) that provides a service for automated kilonova searches. Another example of an "add-on" to existing facilities is the Gamma-ray Urgent Archiver for Novel Opportunities (GUANO) toolkit (Tohuvavohu et al. 2020) that performs low-latency searches for subthreshold/off-axis GRBs and downlinks interesting candidates for deeper searches which would otherwise be lost from traditional search algorithms. Given the rarity of KNe, no individual approach at this stage can serve as the perfect solution and therefore having complementary efforts will be crucial for the future of multi-messenger astrophysics.

\section{ACKNOWLEDGEMENTS}

D. C. is supported by the Illinois Survey Science Fellowship from the Center for AstroPhysical Surveys (CAPS) ${ }^{18}$ at the National Center for Supercomputing Applications (NCSA),

17 https://scimma.org/

18 https://caps.ncsa.illinois.edu/
University of Illinois Urbana-Champaign. D. C. acknowledges computing resources provided by CAPS to carry our this research. This work made use of the Illinois Campus Cluster, a computing resource that is operated by the Illinois Campus Cluster Program (ICCP) in conjunction with NCSA which is supported by funds from the University of Illinois at UrbanaChampaign. This research also used resources of the National Energy Research Scientific Computing Center (NERSC), a U.S. Department of Energy Office of Science User Facility located at Lawrence Berkeley National Laboratory, operated under Contract No. DE-AC02-05CH11231.

D. C. is grateful to Richard Kessler for help with using SNANA. D. C. would like to thank Igor Andreoni for helpful discussions, Alex Gagliano for comments on the draft, Michael Coughlin and Mattia Bulla for help with the BU19 models. The authors specially thank Leo Singer for critical comments. The authors would also like to thank the anonymous referee for helpful comments.

\section{DATA AVAILABILITY}

The SED models used in the study are a part of SNANA (Kessler et al. 2009) package data available at https://zenodo.org/record/4001178\#. YR3qAFt0lcA. The skymaps were generated by running the BAYESTAR (Singer \& Price 2016) code. The neural network architecture used Tensorflow (Abadi et al. 2015) and Keras (Chollet et al. 2015). Currently, the neural network architecture is implemented as a branch of astrorapid in https://github.com/deepchatterjeeligo/astrorapid/ tree/kn-rapid. Eventually, this will be moved to a new public repository. Codes for producing lightcurves from representative SED models in Fig. 3 are avaiable in https://github.com/deepchatterjeeligo/bulla-models. A prototype trained classifier is demonstrated at https://github.com/broker-workshop/tutorials/blob/ main/ANTARES/lsst-broker-workshop-mma-demo. ipynb.

Trained weights and training data for this work will be made available upon request to the authors.

\section{APPENDIX A: ESTIMATING DETECTION EFFICIENCY FOR ZTF}

Here, we show how we estimated the detection efficiency of ZTF: the probability of a source detection for given flux signal-to-noise ratio eff $(S / N)$. We used the magnitude uncertainty $\sigma_{m}$ from the third data release of ZTF (ZTF DR3) ${ }^{19}$ to plot distribution of the number of defections $n(S / N)$ assuming that $S / N=\ln \left(10^{0.4}\right) / \sigma_{m}$. This distribution can be considered as $n(S / N)=n_{\text {tot }} p_{\mathrm{S} / \mathrm{N}}(S / N) \operatorname{eff}(S / N)$ where $n_{\text {tot }}$ is the total number of occurred events, and $p_{\mathrm{S} / \mathrm{N}}(S / N)$ is a probability density function of all (detected and non-detected) events. We assumed that for $10 \lesssim S / N \lesssim 100$ (it roughly corresponds to $21 \gtrsim m \gtrsim 14.5$ for $R$ passband and under an assumption of the Poisson noise) the detection is $100 \%$ effective which means that for this interval $n(S / N) \sim p_{\mathrm{S} / \mathrm{N}}(S / N)$. We found that for considering $S / N$ interval the distribution can be fitted by power-law function $[\beta(S / N)]^{\alpha}$ with exponent

19 http://ztf.caltech.edu/page/dr3 
$\alpha=-1.36$ for $g,-1.30$ for $R$, and -1.53 for $i$. Note that we would have $\alpha=-3 / 2$ for Poisson noise and isotropic distribution of sources of the same luminosity, which is close to found values and allows us to consider that $p_{\mathrm{S} / \mathrm{N}}(S / N) \sim(S / N)^{\alpha}$ can be used for all $S / N$ values. So under this framework eff $=n(S / N) /[\beta(S / N)]^{\alpha}$. For simplicity and to avoid efficiency to be greater $100 \%$ we fitted obtained efficiency curve by a sigmoid function $\left(1+(a /(S / N))^{b}\right)^{-1}$. In this work we aren't interested in detection efficiency for bright objects, therefore we ignore $S / N \gg 10$ when performed the last fit. In the left panel of Fig. A1 we plot normalized $S / N$ distributions and their power-law fits, in its right panel we plot efficiency distribution and their sigmoid fits. Finally, we multiplied $(S / N)$ by the factor of $\sqrt{2}$ to take into account the noise level difference in PSF photometry of ZTF DR3 and differential photometry of the ZTF alert stream under of assumption that image subtraction leads to twice large variance of the source flux estimate.

\section{APPENDIX B: EXAMPLE LIGHTCURVES}

In Fig. B1 we show a few randomly chosen example lightcurves from the training set used in this work.

\section{APPENDIX C: VARIATION OF $M_{\mathrm{ej}}$ WITH EOS}

The ejecta mass in Eq. (1) can be a strong function of the stiffness of the NS. Stiffer stars allow for larger radii at the same mass, and therefore are easily tidally disrupted. However, the tidal effects from GW170817 constrains several of the EoSs in the literature. Recently, Ghosh et al. (2021) have performed model selection of representative EoSs against tidal deformability measurements of GW170817. In Fig. C1, we restrict to those that have the highest evidences, and assess the variation of the ejecta mass from the DU17 relation. We sample, component masses uniformly $\in[1,3] M_{\odot}$, rejecting combinations in case they are greater than the maximum mass allowed by an EoS. We find that the distribution of the ejecta mass is not significantly affected in this case. Hence, our choice of using APR4_EPP is justified. The model grids used in this work have an spacing of $\mathcal{O}\left(10^{-2}\right)$ in ejecta mass which is large compared to the differences in ejecta masses from the strongly supported EoSs.

\section{APPENDIX D: RESULTS FROM LVK DESIGN SENSITIVITY}

In this section, we repeat the training using a dataset set where we use the same objects but consider a designsensitivity noise-curve for LIGO/Virgo/KAGRA. We also restrict to only events that are detected in at least 3-detectors. In Fig. D1 and Fig. D2, we show the performance of this classifier on AT 2017gfo and AT 2019npv as in Fig. 8 and Fig. 9 in the main body of the text. We find that the results are not strongly sensitive to the change in detector sensitivity.

\section{REFERENCES}

Abadi M., et al., 2015, TensorFlow: Large-Scale Machine Learning on Heterogeneous Systems, https://www.tensorflow.org/

Abbott B., et al., 2016, Physical Review Letters, 116

Abbott B., et al., 2017a, Physical Review Letters, 119

Abbott B. P., et al., 2017b, Nature, 551, 85

Abbott B. P., et al., 2017c, ApJ Lett., 848, L12

Abbott B. P., et al., 2018a, Living Reviews in Relativity, 21, 3

Abbott B. P., et al., 2018b, Phys. Rev. Lett., 121, 161101

Abbott R., et al., 2020a, arXiv e-prints, p. arXiv:2010.14527

Abbott R., et al., 2020b, The Astrophysical Journal, 896, L44

Andreoni I., et al., 2020, The Astrophysical Journal, 890, 131

Andreoni I., et al., 2021, Fast-transient Searches in Real Time with ZTFReST: Identification of Three Optically-discovered Gamma-ray Burst Afterglows and New Constraints on the Kilonova Rate (arXiv:2104.06352)

Antier S., et al., 2019, Monthly Notices of the Royal Astronomical Society, 492, 3904-3927

Arcavi I., et al., 2017, Nature, 551, 64

Arun K. G., Iyer B. R., Sathyaprakash B. S., Sundararajan P. A., 2005, Phys. Rev. D, 71, 084008

Bai S., Zico Kolter J., Koltun V., 2018, arXiv e-prints, p. arXiv:1803.01271

Bellm E. C., et al., 2019, PASP, 131, 018002

Blinnikov S. I., Novikov I. D., Perevodchikova T. V., Polnarev A. G., 1984, Soviet Astronomy Letters, 10, 177

Bulla M., 2019, MNRAS, 489, 5037

Chatterjee D., Nugent P. E., Brady P. R., Cannella C., Kaplan D. L., Kasliwal M. M., 2019, The Astrophysical Journal, 881, 128

Chollet F., et al., 2015, Keras, https://keras.io

Coughlin M. W., 2020, Nature Astronomy, 4, 550

Coughlin M. W., et al., 2020, Nature Communications, 11, 4129

Coulter D. A., et al., 2017, Science, 358, 1556

Dietrich T., Ujevic M., 2017, Classical and Quantum Gravity, 34, 105014

Dobie D., et al., 2019, The Astrophysical Journal, 887, L13

Feindt U., Nordin J., Rigault M., Brinnel V., Dhawan S., Goobar A., Kowalski M., 2019, Journal of Cosmology and Astroparticle Physics, 2019, 005-005

Foley R. J., Mandel K., 2013, ApJ, 778, 167

Gagliano A., Narayan G., Engel A., Kind M. C., 2021, The Astrophysical Journal, 908, 170

Ghosh S., Liu X., Creighton J., Kastaun W., Pratten G., Magana Hernandez I., 2021, arXiv e-prints, p. arXiv:2104.08681

Goldstein A., et al., 2017, ApJ, 848, L14

Goldstein D., Andreoni I., Hankins M., et al., 2019, GCN, 25393

Guy J., et al., 2007, Astronomy \& Astrophysics, 466, 11-21

He K., Zhang X., Ren S., Sun J., 2015, arXiv e-prints, p. arXiv:1512.03385

Herner K., Palmese A., Soares-Santos M., et al., 2019, GCN, 25398

Hlozek R., et al., 2020, Results of the Photometric LSST Astronomical Time-series Classification Challenge (PLAsTiCC) (arXiv:2012.12392)

Ho A. Y. Q., et al., 2018, The Astrophysical Journal, 854, L13

Holz D. E., Hughes S. A., 2005, ApJ, 629, 15

Ivezic Z., et al., 2019, The Astrophysical Journal, 873, 111

Kasdin N. J., et al., 2020, Space Telescopes and Instrumentation 2020: Optical, Infrared, and Millimeter Wave

Kasen D., Metzger B., Barnes J., Quataert E., Ramirez-Ruiz E., 2017, Nature, 551, 80-84

Kasliwal M. M., et al., 2017, Science, 358, 1559

Kessler R., et al., 2009, PASP, 121, 1028

Kessler R., Conley A., Jha S., Kuhlmann S., 2010a, Supernova Photometric Classification Challenge (arXiv: 1001.5210)

Kessler R., et al., 2010b, ApJ, 717, 40

Kessler R., et al., 2019, PASP, 131, 094501 



Figure A1. Signal-to-noise distributions. Left panel: The distribution of ZTF DR3 detections over $S / N$ normalized to have have unity integral (solid lines). Thin dashed lines show power-law fits for these distributions for the interval $6<S / N<80$, corresponding exponents $\alpha$ are listed in the legend. Right panel: The detection efficiency distribution. Solid lines show the ratio of $S / N$ distributions to corresponding fits (solid to dashed lines from the left panel). Dashed lines show $\left(1+(a /(S / N))^{b}\right)^{-1}$ fits of these distributions, fit parameters are given in the legend.
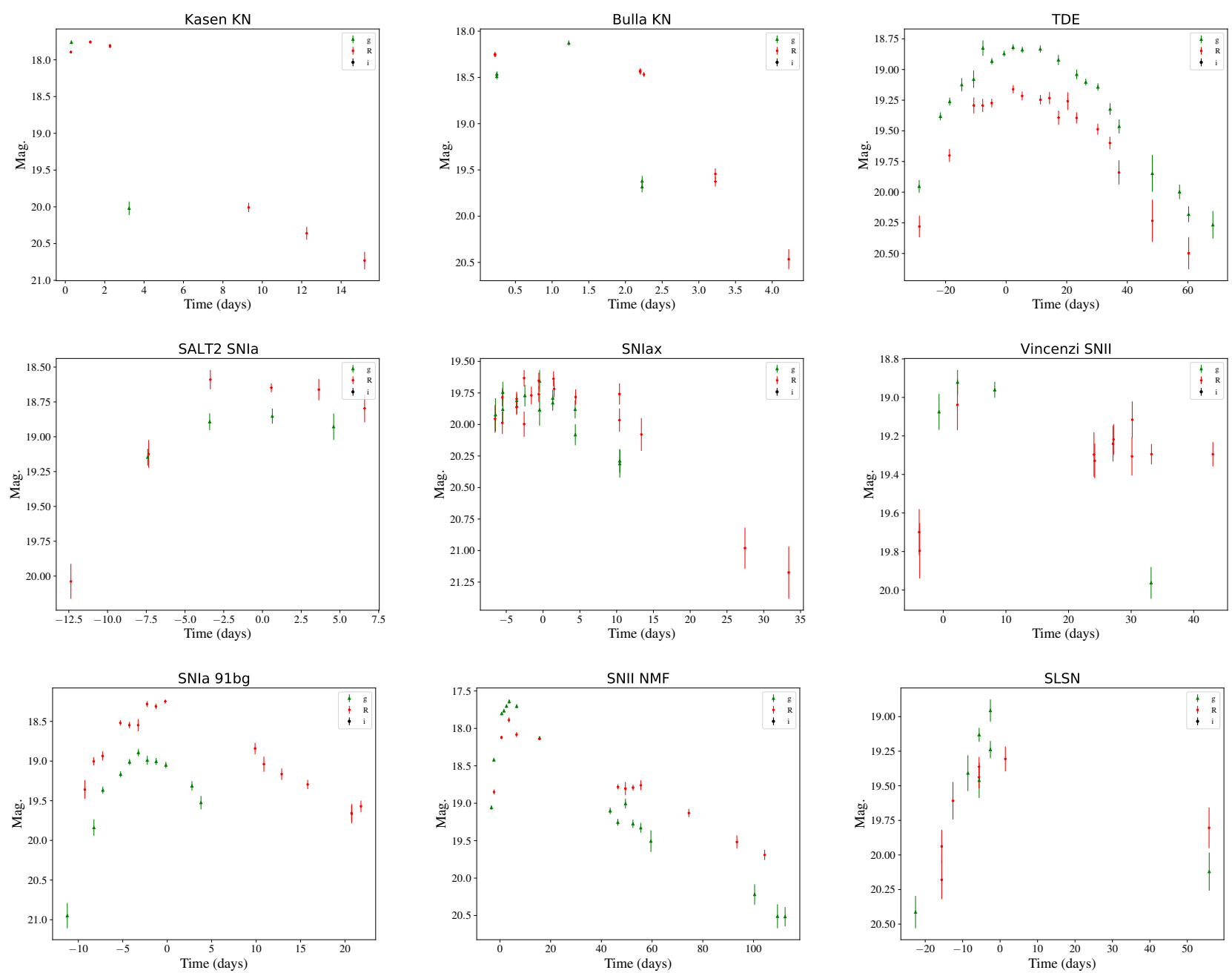

Figure B1. A random sample of example lightcurves from the training set 


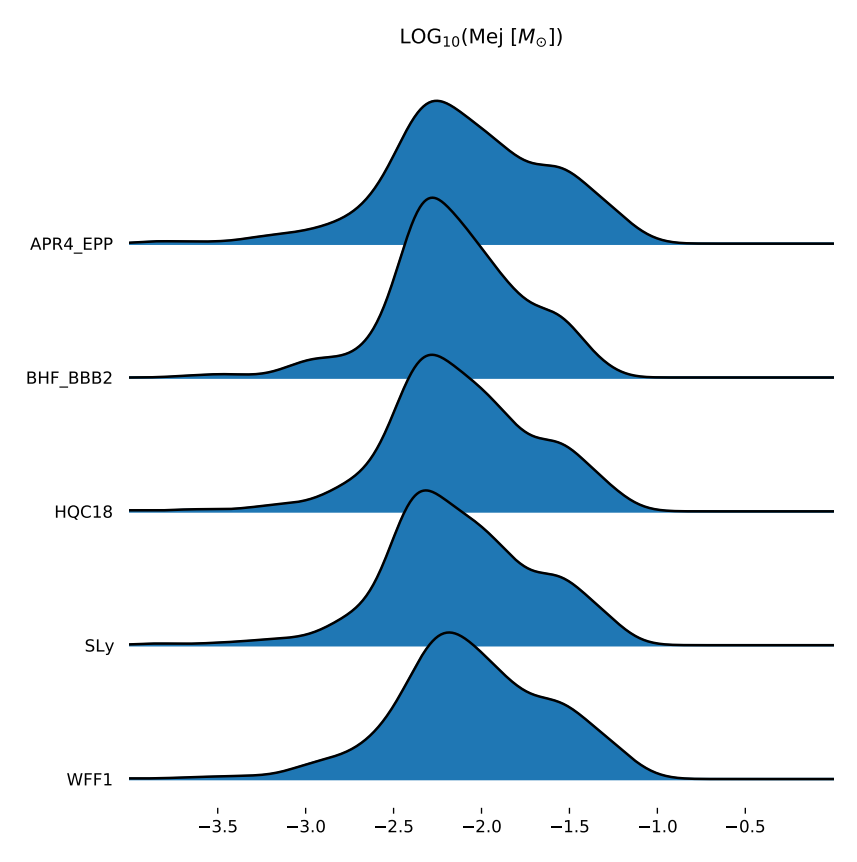

Figure C1. This figure shows the distribution of the variation of ejecta mass, $M_{\mathrm{ej}}$, with EoS.

LIGO Scientific Collaboration, Virgo Collaboration 2019, GCN, 25333

Lattimer J. M., Schramm D. N., 1974, ApJ, 192, L145

Lattimer J. M., Schramm D. N., 1976, ApJ, 210, 549

Li L.-X., Paczyński B., 1998, ApJ, 507, L59

Lipunov V. M., et al., 2017, The Astrophysical Journal, 850, L1

Lochner M., McEwen J. D., Peiris H. V., Lahav O., Winter M. K., 2016, ApJS, 225, 31

Martínez-Palomera J., Bloom J. S., Abrahams E. S., 2020, arXiv e-prints, p. arXiv:2005.07773

Matheson T., et al., 2021, AJ, 161, 107

Metzger B. D., et al., 2010, MNRAS, 406, 2650

Miller M. C., et al., 2019, ApJ, 887, L24

Morgan R., et al., 2020, The Astrophysical Journal, 901, 83

Muthukrishna D., Narayan G., Mandel K. S., Biswas R., Hložek R., 2019, PASP, 131, 118002

Narayan G., et al., 2018a, ApJS, 236, 9

Narayan G., et al., 2018b, ApJS, 236, 9

Radice D., Perego A., Hotokezaka K., Fromm S. A., Bernuzzi S., Roberts L. F., 2018, The Astrophysical Journal, 869, 130

Remy P., 2020, Temporal Convolutional Networks for Keras, https://github.com/philipperemy/keras-tcn

Schutz B. F., 1986, Nature, 323, 310

Shibata M., Hotokezaka K., 2019, Annual Review of Nuclear and Particle Science, 69, 41-64

Singer L. P., Price L. R., 2016, Phys. Rev. D, 93, 024013

Smartt S., Smith K. W., Srivastav S., et al., 2019, GCN, 25455

Soares-Santos M., et al., 2017, The Astrophysical Journal, 848, L16

Stachie C., Coughlin M. W., Christensen N., Muthukrishna D., 2020, Monthly Notices of the Royal Astronomical Society, 497, $1320-1331$

Street R. A., Bowman M., Saunders E. S., Boroson T., 2018, in Guzman J. C., Ibsen J., eds, Society of Photo-Optical Instrumentation Engineers (SPIE) Conference Series Vol. 10707, Software and Cyberinfrastructure for Astronomy V. p. 1070711 (arXiv: 1806.09557), doi:10.1117/12.2312293

Street R. A., et al., 2020, in Society of Photo-Optical Instrumentation Engineers (SPIE) Conference Series. p. 1144925, doi:10.1117/12.2559986

T.-W. Chen T. Schweyer A. N. G., et al., 2019, GCN, 25457

Tanvir N. R., Levan A. J., Fruchter A. S., Hjorth J., Hounsell R. A., Wiersema K., Tunnicliffe R. L., 2013, Nature, 500, 547-549

Tanvir N. R., et al., 2017, The Astrophysical Journal, 848, L27

Tohuvavohu A., Kennea J. A., DeLaunay J., Palmer D. M., Cenko S. B., Barthelmy S., 2020, The Astrophysical Journal, 900, 35

V. Lipunov D. Vlasenko E. G., et al., 2019, GCN, 25474

Valenti S., et al., 2017, The Astrophysical Journal, 848, L24

Vieira N., et al., 2020, The Astrophysical Journal, 895, 96

Vincenzi M., Sullivan M., Firth R. E., Gutiérrez C. P., Frohmaier C., Smith M., Angus C., Nichol R. C., 2019, Monthly Notices of the Royal Astronomical Society, 489, 5802-5821

Watson A. M., et al., 2020, Monthly Notices of the Royal Astronomical Society, 492, 5916-5921

X. Wang S. Antier M. C., et al., 2019, GCN, 25485

de Wet S., et al., 2021, Astronomy \& Astrophysics, 649, A72

This paper has been typeset from a $\mathrm{T}_{\mathrm{E}} \mathrm{X} / \mathrm{LAT}_{\mathrm{E}} \mathrm{X}$ file prepared by the author. 

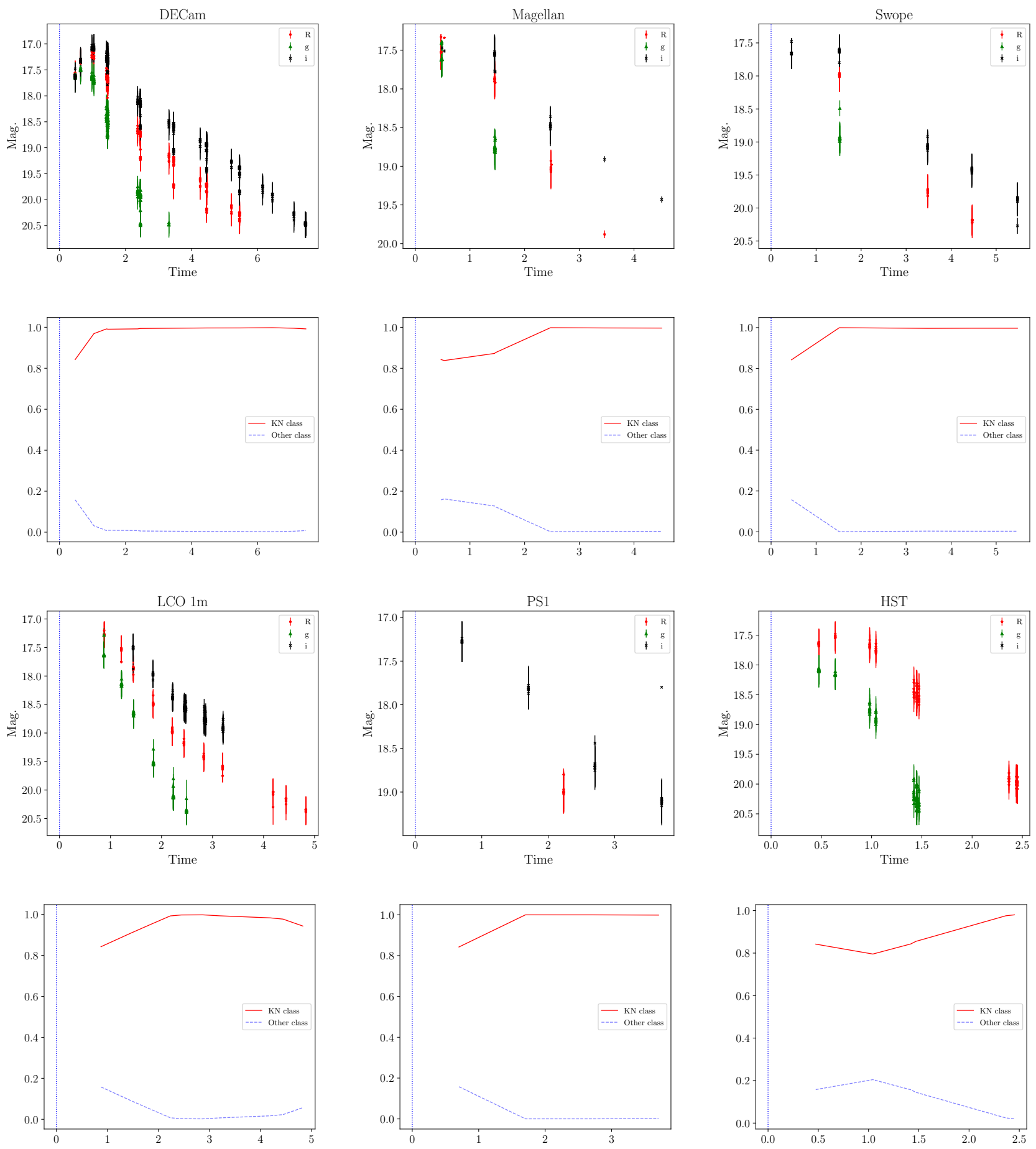

Figure D1. Similar to Fig. 8 except the the classifier trained on design sensitivity era on $\sim 1$ week data for GW170817 obtained from the Open Kilonova catalog. 

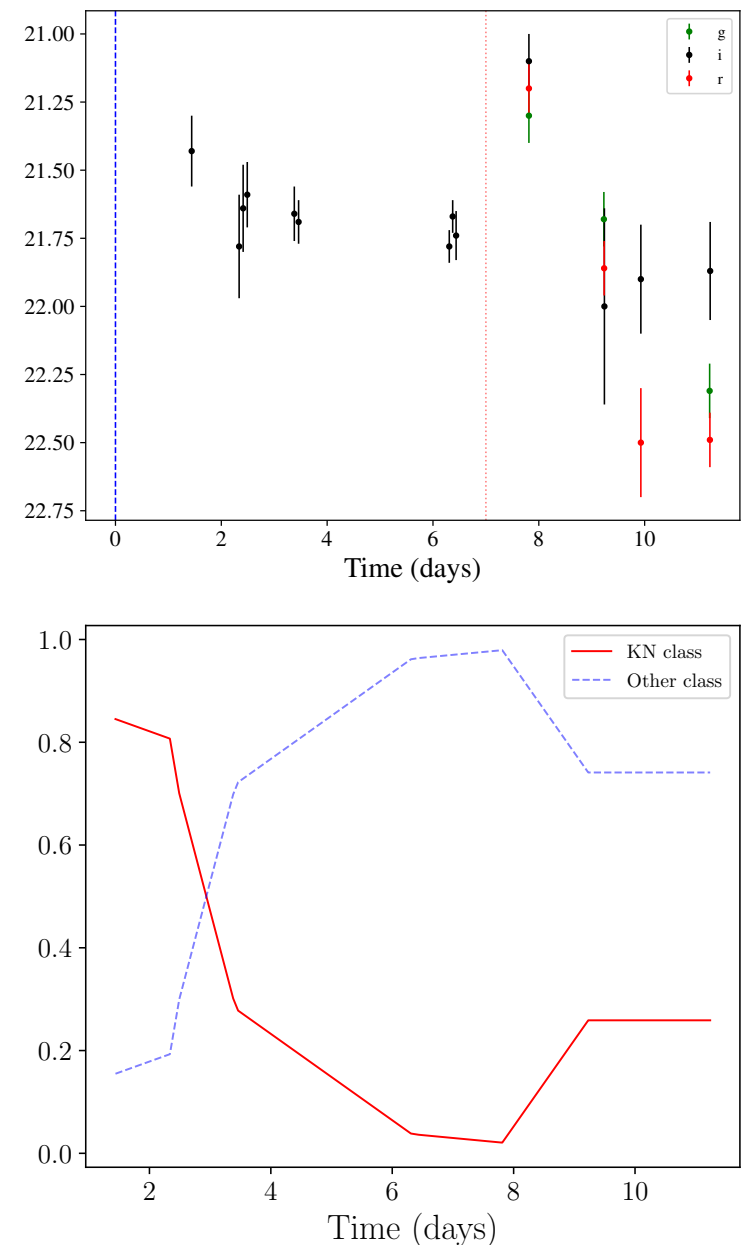

Figure D2. AT 2019npv classification similar to Fig. 9, except the classifier is trained on simulations in the design sensitivity era. We find that the results are robust irrespective of the noise-curve between $\mathrm{O} 4$ and design sensitivity. 